\title{
Numerical analysis of the gas-particle two-phase flow in a multistep dust collector
}

\author{
Jiafeng Yao ${ }^{1,2, *}$, Zheng Wang ${ }^{1}$, Minghao $\mathrm{Yu}^{3}$, Yaoyao Wang ${ }^{1}$, Bai Chen ${ }^{1}$ and \\ Hongtao $\mathrm{Wu}^{1,2}$ \\ ${ }^{1}$ College of Mechanical and Electrical Engineering, Nanjing University of Aeronautics and Astronautics, Nanjing, 210016, China \\ ${ }^{2}$ Nan Ma Intelligent Manufacturing Research Institute Limited Company, MA'Anshan, 243000, China \\ ${ }^{3}$ Faculty of Mechanical and Precision Instrument Engineering, Xi'an University of Technology, Xi'an 710048, China
}

\begin{abstract}
The gas-particle two-phase flow in a multistep dust collector has been numerically analysed for the improvement of particle separation efficiency under several typical industrial conditions. Moreover, the calculated results are validated by experiments with a maximum error $e_{\max }=4.6 \%$. The results indicate the influence of the geometrical dimensions of the multistep dust collector on particle separation efficiency and pressure drop. The present study proposes an effective approach to optimize a dust collector, which can improve particle separation efficiency at low cost and in a short development cycle.
\end{abstract}

Keywords: Gas-particle, multistep dust collector, numerical analysis, pressure drop, separation efficiency.

WITH the rapid economic development, global industrialization and urbanization, the problem of air pollution in industrial cities is attracting more attention. Air pollution makes human beings susceptible to diseases like tuberculosis and lung cancer. To purify the polluted air, cyclone collectors are widely used in industrial processes for the separation of dust from gas streams to reduce air contaminants ${ }^{1-3}$. The popularity of such collectors is due to their simplicity and the fact that they consume less energy as well as the low cost in construction, operation and maintenance $e^{4-6}$. According to the entrance structure, the cyclone collector can be divided into two types - cyclone separator and axial flow separator.

The cyclone collector geometry has been almost the same for over a century. Particles of different size can be collected using the two types of collectors. Generally, the cyclone separator can collect particles under $20 \mu \mathrm{m}$ in size, which is not enough in specific industrial applications. The axial flow separator can remove particles smaller than $d_{\mathrm{p}}=10 \mu \mathrm{m}$ from solid-gas flows. The combination proposed in the present study can improve the separation efficiency of smaller sized particles ${ }^{7}$. Extensive studies have been conducted on the two types of cyclone collectors. Xue et al. $^{8}$ showed that particle concentration was low in the inner region $(r / R \leq 0.75)$ and

\footnotetext{
*For correspondence. (e-mail: jiaf.yao@nuaa.edu.cn)
}

increased greatly in the outer region $(r / R \geq 0.75)$ of a gas cyclone separator. They also proposed that the particle separation ability of the separator was weakened with rise in temperature. Hsiao et al. ${ }^{9,10}$ studied eight different geometric configurations of the axial flow separator when particle size changed from $d_{\mathrm{p}}=272 \mathrm{~nm}$ to $d_{\mathrm{p}}=448 \mathrm{~nm}$, where $p$ is the diameter of particle size. They mainly examined the effects of the upside-down cup, vortex finder (VF) length, and cyclone base geometry on the particle collection efficiency. However, they only validated the applicability of the optimal model that they proposed for the axial flow separator. Chen and Tsai ${ }^{11}$ studied the mini geometry of axial flow separator and found that it can prominently separate particles smaller than $10 \mu \mathrm{m}$; even nanoparticles when the inlet velocity is high enough.

Numerical simulations are extensively conducted for geometries factors of the cyclone collectors. However, there are not many numerical studies on the combination of the two types of cyclone collectors. In fact, both the cyclone collectors have critical defects when they work alone. The total efficiency of the tangential cyclone collector is still less than $70 \%$ (ref. 12).

Next, we proposed the concept of an industrial-scale multistep dust collector by combining a cyclone separator, an axial flow separator and a filter. We expect to enhance the performance of cyclone collector. The flow field of two types of cyclone separators was simulated using shear-stress transport model (SST). Effect of geometrical dimensions on particle separation efficiency and pressure drop are also discussed. Moreover, the numerical results are validated with experimental data to evaluate the reliability of the simulation results.

\section{Theory and conditions \\ Construction of the cyclones collector}

The multistep dust collector consists of two kinds of cyclone collectors. As a first step, the gas-particle flow enters into a cyclone separator (I in Figure 1). The diameter of the inlet is $D_{\text {in }}=0.35 D$, where $D$ is the diameter of the axial flow cyclone pipe. The hopper is not higher than $2 D$, though a long tapered body is intended to make the 


\section{RESEARCH ARTICLES}

collection of fine particles more easy. However, this added length may enhance pressure drop and create problems with installation space requirements as well as high material costs. The processed gas-particle flow mainly containing tiny particles exits from the top of the separator through the outlet pipe. The second step is an axial flow separator with an eight-blade fan (II in Figure 1). The diameter of the fan is $D_{\mathrm{f}}=0.5 D$, where $D$ is the inlet diameter. Blade length $L_{2}=0.5 D$ and outlet diameter $D_{3}=0.6 D$. A diversion-cone is installed behind the fan to increase the rotation of the gas flow, and its diameter is $D_{\mathrm{C}}=0.5 \mathrm{D}$. Another collector is placed below the outlet pipe for collecting particles which collide with a horizontal wall. The clean flow leaves the outlet region and is then filtered by a filter screen. Since modelling of a filter will make the simulation more complex, we did not consider a filter in the model. Another more reason is that after the two steps of separation, only a few particles reach the filter. This result has been proved by experiments.

The function of the blade can be represented as follows

$$
\begin{aligned}
& x=(1-\cos \theta) / \pi, \\
& y=-\sin (\pi t-\sin \theta), \\
& z=-(1-\cos (\pi t-\sin \theta)) .
\end{aligned}
$$

\section{Turbulent model}

The SST model is built for accurate prediction of aeronautics flows with strong adverse gradients and separation. Any suitable model should be extensible to modern

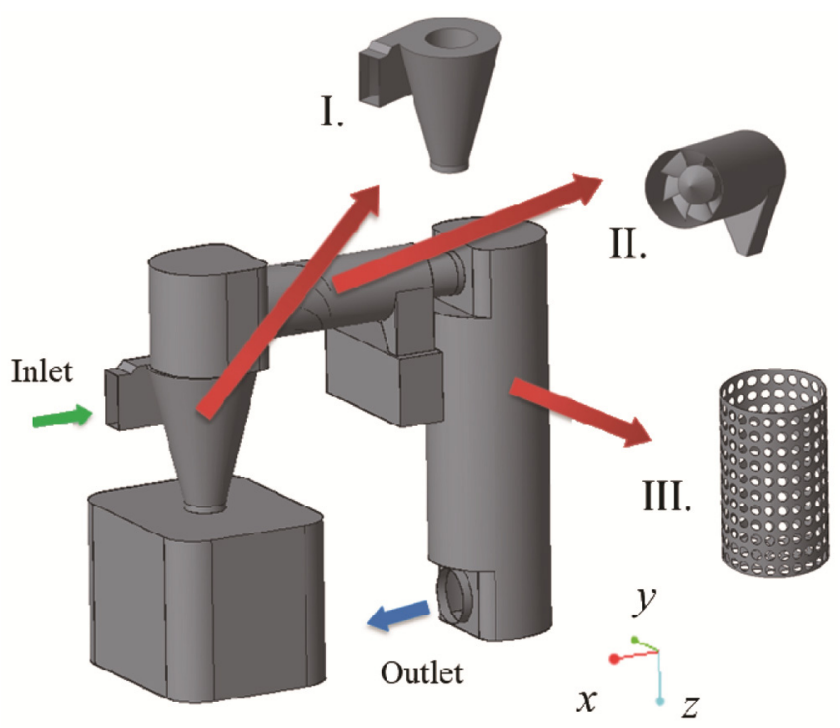

Figure 1. Geometry of the multistep dust collector. I, Cyclone separator; II, Axial flow separator and III, Filter. three-dimensional Navier-Stokes codes due to its algebraic formulation ${ }^{13}$. The $k-\varepsilon$ model is not able to capture the proper behaviour of turbulent boundary layers up to separation. The $k-\omega$ model is substantially more accurate than the $k-\varepsilon$ model for the location near the wall layers, and has therefore been successful for flows with moderate adverse pressure gradients, but fails for flows with pressure-induced separation ${ }^{14}$. The SST model is the transformation of $k-\varepsilon$ and $k-\omega$ in different positions by changing the coefficient $\theta_{k-\varepsilon}$ and $\varphi_{k-\omega}$.

$$
\phi_{\mathrm{sst}}=\theta_{k-\varepsilon} F_{1}+\phi_{k-\omega}\left(1-F_{1}\right) .
$$

The complete formulation of the SST model is given below.

$$
\begin{aligned}
& \frac{\partial \rho k}{\partial t}+\frac{\partial \rho U_{i} k}{\partial x_{i}}=\tilde{P}_{k}-\beta^{*} \rho k \omega+\frac{\partial}{\partial x_{i}}\left[\left(v+\sigma_{k} v_{t}\right) \frac{\partial k}{\partial x_{i}}\right], \\
& \frac{\partial \rho \omega}{\partial t}+\frac{\partial \rho U_{i} \omega}{\partial x_{i}}=\alpha \rho S^{2}-\beta \rho \omega^{2} \\
& +\frac{\partial}{\partial x_{i}}\left[\left(v+\sigma_{\omega} v_{t}\right) \frac{\partial \omega}{\partial x_{i}}\right]+2\left(1-F_{1}\right) \rho \sigma_{\omega^{2}} \frac{1}{\omega} \frac{\partial k}{\partial x_{i}} \frac{\partial \omega}{\partial x_{i}} .
\end{aligned}
$$

The blending function $F_{1}$ is defined by

$$
F_{1}=\tanh \left\{\left\{\min \left[\max \left\{\frac{\sqrt{k}}{\beta^{*} \omega y^{\prime}}, \frac{500 v}{y^{2} \omega}\right\}, \frac{4 \sigma_{\omega^{2}} k}{C_{d, k \omega} y^{2}}\right]\right\}^{4}\right\},
$$

$$
C_{d, k \omega}=\max \left\{2 \rho \sigma_{\omega^{2}} \frac{1}{\omega} \frac{\partial k}{\partial x_{i}} \frac{\partial \omega}{\partial x_{i}}, 10^{-10}\right\}
$$

where $y$ is the distance to the nearest wall. $F_{1}$ is zero away from the surface, and approaching to 1 inside the boundary layer.

The turbulent eddy viscosity is defined as follows

$$
v_{t}=\frac{\alpha_{1} k}{\max \left\{\alpha_{1} \omega, \mathrm{SF}_{2}\right\}}
$$

where $S$ is the invariant measure of strain rate and $F_{2}$ is a second blending function defined by

$$
F_{2}=\tan h\left(\max \left\{\frac{2 \sqrt{k}}{\beta^{*} \omega y}, \frac{500 v}{y^{2} \omega}\right\}\right)^{2} .
$$

All constants are computed by the $k-\varepsilon$ and $k-\omega$ models. The constants for the SST model are: $\beta^{*}=0.09, \alpha_{1}=5 / 9$, 
$\beta_{1}=3 / 40, \sigma_{k 1}=0.85, \sigma_{\omega 1}=0.5, \alpha_{2}=0.44, \beta_{2}=0.0828$, $\sigma_{k 2}=1, \sigma_{\omega 2}=0.856$ (ref. 13).

\section{Gas-particle two-phase flow model}

The inlet wood solids concentration is $C_{\mathrm{i}}=0.005 \mathrm{~kg} / \mathrm{m}^{3}$ and the corresponding particle phase volume fraction is smaller than $\varepsilon=5 \%$. Except near the wall region, the solids concentration distribution in most of volume cyclone can be calculated using the Lagrangian approach without considering the particle interaction, which is called the discrete phase model (DPM) in computational fluid dynamics (CFD) ${ }^{4}$.

The momentum equation of a particle in the two-phase flow is expressed as

$$
\begin{aligned}
& \frac{\mathrm{d} u_{p}}{\mathrm{~d} t}=\frac{1}{\tau}\left(u_{g}+u_{g}^{\prime}-u_{p}\right)-g, \\
& \frac{\mathrm{d} v_{p}}{\mathrm{~d} t}=\frac{1}{\tau}\left(v_{g}+v_{g}^{\prime}-v_{p}\right)+\frac{w_{p}^{2}}{r_{0}}, \\
& \frac{\mathrm{d} w_{p}}{\mathrm{~d} t}=\frac{1}{\tau}\left(w_{g}+v_{g}^{\prime}-w_{p}\right)-\frac{u_{p} w_{p}}{r_{0}} .
\end{aligned}
$$

Here $p$ and $g$ are particles and gas phase, $u$ is the fluid phase velocity, $u_{g}^{\prime}, v_{g}^{\prime}, w_{g}^{\prime}$ are the fluctuation velocity components, and $\tau$ is the relaxation time of particles ${ }^{15}$.

$$
\tau=\frac{\rho_{p} d^{2} p}{18 \mu} \frac{24}{C_{D} R e_{p}} .
$$

The particle separation efficiency $\varepsilon_{\mathrm{p}}$ is calculated by

$$
\varepsilon_{p}=\frac{m_{c} f_{c} \Delta D_{p}}{m_{c} f_{c} \Delta D_{p}+m_{s} f_{s} \Delta D_{p}} .
$$

The particle size distributions are set with constant value. There are several different particle sizes $\left(d_{\mathrm{P}}=1,3,5,8\right.$, $10,20,30,40$ and $50 \mu \mathrm{m})$. The number of each particle type is 10,000 .

Boundary condition: The velocity at the inlet is $v_{\text {in }}=22 \mathrm{~m} / \mathrm{s}$. The pressure at the outlet is $P_{\text {out }}=0 \mathrm{~Pa}$ of the static pressure. Air enters the inlet with boundary temperature of $300 \mathrm{~K}$. The flow is assumed to be fully developed at the inlet and outlet. The walls and canes are considered to be no-slip. The perpendicular coefficient of restitution of the collector is zero. Particles entering into the collector and not coming out will be considered as collected on the walls. The material of the particles is hardwood and the density is $\rho_{\mathrm{w}}=800 \mathrm{~kg} / \mathrm{m}^{3}$ while concentration of inlet face is $\rho_{\mathrm{in}}=5 \mathrm{~g} / \mathrm{m}^{3}$.

\section{Results and discussion}

\section{Effect of multistep dust collector}

Particle separation efficiency of the multistep dust collector was compared according to varied particle diameters (Figure $2 a$ ). The separation efficiency was enhanced using multistep cyclone collector (I + II) compared with a individual collector (I or II). $\varepsilon_{\mathrm{p}}$ of the multistep cyclone collector was $12 \%$ higher than the cyclone separator with particle diameter below $d_{\mathrm{p}}=20 \mu \mathrm{m}$, while the pressure drop $\Delta P$ only increased by $10 \%$. The separation performance of the multistep dust collector was better than the axial flow separator. In general, as the diameter increases, the separation efficiency increases; but in this condition, separation efficiency decreases at $30 \mu \mathrm{m}$ diameter. Maybe the particles interaction increased quickly in this size. More study will be conducted to explain the above phenomenon in the next study.

\section{Effect of blade number}

The separation efficiency is also influenced by the number of blades in the axial flow separator. More blades lead to a higher pressure drop and fewer blades induce a lower separation efficiency (Figure $2 b$ ). $\varepsilon_{\mathrm{p}}$ reaches a maximum value when $N=8$. With the increase in blade number, the performance of the multistep dust collector improves at the range of $N=6-8$. The similar results have been studied by Houlin et al. ${ }^{16}$. Particles need enough momentum to enter into their maximum spiral orbit, because centrifugal force is affected by acceleration. Particles are more likely to escape from the exit because the orbit radius is too small when $N=6$. The situation of $N=7$ is not good enough in the separation effort of $d_{\mathrm{P}}$ below $10 \mu \mathrm{m}$. There is no difference between $N=8$ and $N=9$. The results show that eight blades is the best choice in this situation.

\section{Effect of some critical geometrical dimensions}

In the axial flow cyclone collector, the dimensions of $L_{1}, L_{2}, D_{3}$ influence the particle separation efficiency $\varepsilon_{\mathrm{p}}$ (Figure 3).

Effect of blade installation site: The velocity distribution on the chamber of the axial flow separator can be adjusted by different blades installation sites $L_{1}$. The collision between particles and flow baffle is greater when more particles enter in the separator ${ }^{17}$. As shown in Figure 4 , the velocity distributions are colour changes on the plane of $y=0$ due to different blade installation positions, i.e. $L_{1}=0.3 D, 0.6 D, 0.7 D$ and $0.75 D$. In the space between blade and outlet pipe, it turned from yellow to red to a deep with the change in $L_{1}$. 

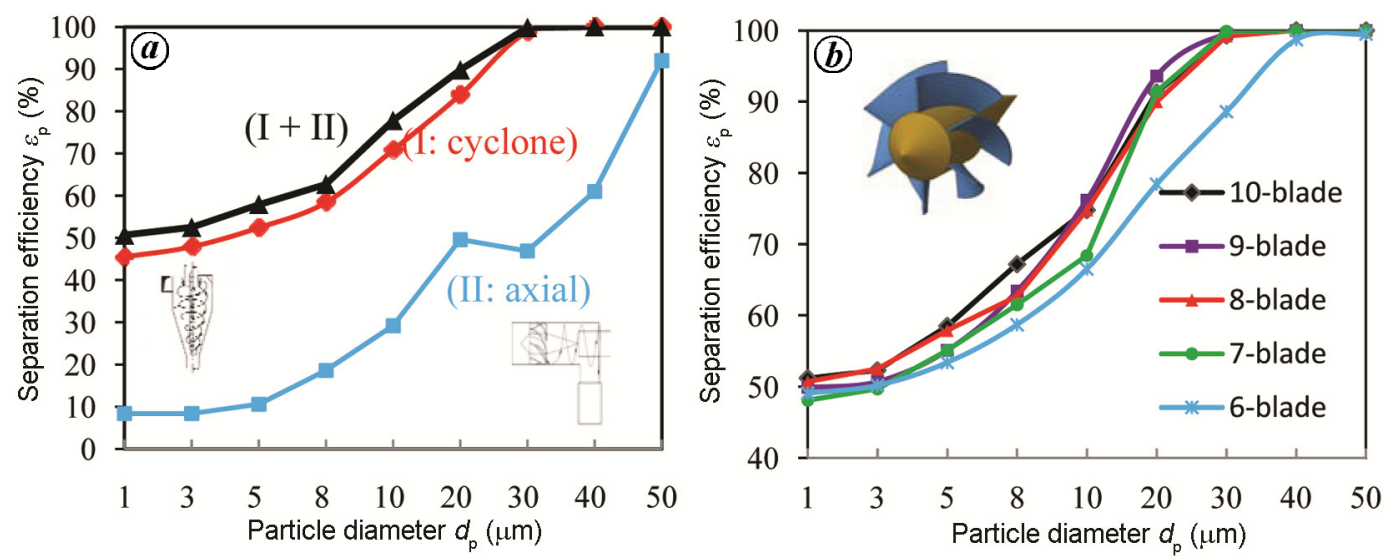

Figure 2. Performance of the multistep dust collector with varied particle diameter, $d_{\mathrm{p}}$. $\boldsymbol{a}$, Comparison of the cyclone and axial flow collector. $\boldsymbol{b}$, Influence of blade number on particle separation efficiency for the axial flow collector.

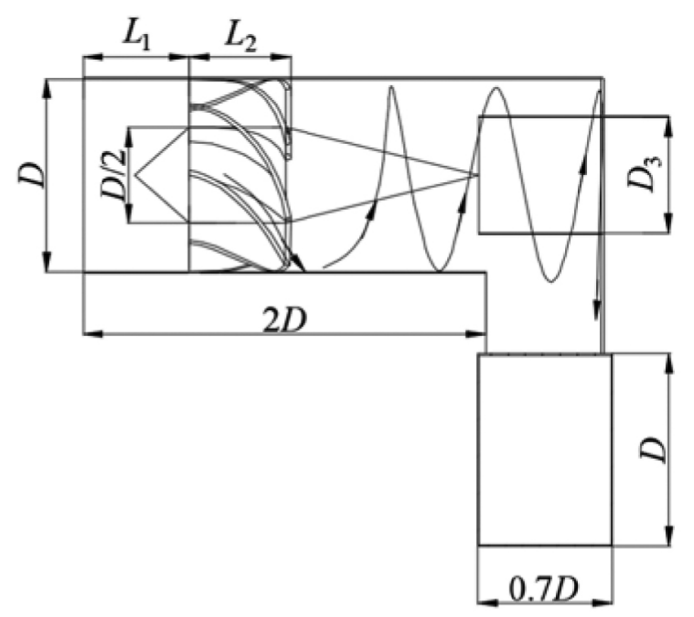

Figure 3. Definition of geometrical dimensions of the axial flow separator. $L_{1}$ is the installation distance of the blade from inlet; $L_{2}$ is the length of the blade; $D$ is the diameter of the inlet; $D_{3}$ is the diameter of the outlet.

As shown in Figure $5 a$, as the value of $L_{1}$ increases from $0.3 D$ to $0.7 D, \varepsilon_{\mathrm{p}}$ increases progressively to about $6 \%$. The energy loss of the gas-particle flow firstly occurs at the terminal of the diversion cone in the chamber. After the swirling flow in the central of the outlet, the energy loss occurs again according to the velocity distribution. Friction between rapid flow and geometry results in momentum loss ${ }^{18}$, which influences the energy exchange of flow baffle and particles. The smaller $L_{1}$ indicates particles moving greater distance after acceleration by blades and energy of flow lost is more when $L_{1}$ is below $0.7 \mathrm{D}$.

$\varepsilon_{\mathrm{p}}$ decreases $4 \%$ when $L_{1}$ is $0.75 D$. The velocity profile of $L_{1}=0.75 \mathrm{D}$ in Figure 4 shows that the gas-particle flow is more rapid compared to other values of $L_{1}$. But the advantage of rapid velocity is not applicable to all situations. The smaller $L_{1}$ indicates that particles are moving further after acceleration by blades and the energy loss of the flow is more when $L_{1}$ is below $0.7 D$. Energy loss is superimposed and static pressure at the outlet is decreased. In addition, as the distance between the blade and the outlet exceeds the limit, the particles accelerated by the blade fall on the inner surface of the outlet pipe along its constant trajectory curve, and finally escape from the cyclone separator. Compared with $L_{1}$ less than $0.75 D$, these phenomena reduce epsilon $p$.

Effect of blade length: The blade length $L_{2}$ will influence the accelerating particles. One of the common characteristics of the axial flow separator is that the faster the particles move in the chamber, the higher is the efficiency. Blade length should be less than a specific dimension to avoid wastage of material.

Figure $5 b$ shows different $\varepsilon_{\mathrm{p}}$ values for simulation with several typical $L_{2}$ values. When $L_{2}$ is greater than $0.45 D$, it has the same epsilon $p$, that is, $L_{2}$ should not exceed this dimension. $\varepsilon_{\mathrm{p}}$ of $L_{2}=0.4 D$ is apparently poor when particle diameter is above $10 \mu \mathrm{m}$. Thus the length of the blade is selected as $L_{2}=0.45 \mathrm{D}$.

Effect of outlet dimension: The obtained results revealed that different axial flow outlet diameters $\left(D_{3}\right)$ have an influence on $\varepsilon_{\mathrm{p}}$ and $\Delta P$. As shown in Figure $5 c$, in practice, the $\varepsilon_{\mathrm{p}}$ of smaller particles can obviously be influenced by varying the outlet diameter of the axial flow separator. Compound effects of centrifugal force and gravity acting on different sized particles make them move forward at different orbits in the chamber. The value of $D_{3}$ should be close to the critical orbit diameter, which is the minimum size particle collected by the axial flow separator. From the numerical study, $\varepsilon_{\mathrm{p}}$ of the cyclone collector is the same when $D_{3}=0.6 D$ or $0.7 D$. $\varepsilon_{\mathrm{p}}$ decreases when $D_{3}$ exceeds $0.7 D$. Thus $D_{3}=0.7 D$ is the optimum value for both $\varepsilon_{\mathrm{p}}$ and $\Delta P$.

According to the numerical study, since the calculated deviation value of $\Delta P_{\mathrm{a}}$ is the same, there is a linear relationship between $\Delta P_{\mathrm{a}}$ and $D_{3}$. The outlet diameter is directly related to the local pressure loss. Figure $5 d$ shows 
Velocity $v$

$$
\begin{aligned}
& \mathrm{m} / \mathrm{s} \\
& 15 \\
& 13 \\
& 11 \\
& 9 \\
& 7 \\
& 5 \\
& 3 \\
& 1
\end{aligned}
$$

Velocity $v$

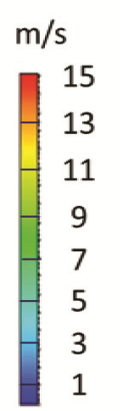

(a)

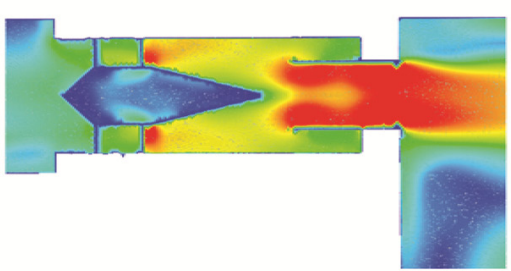

(c)

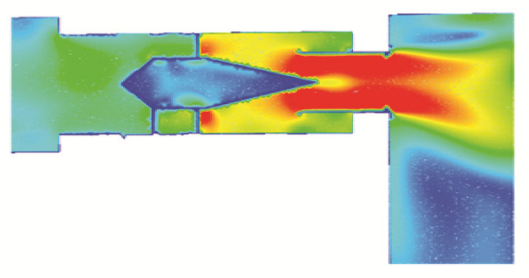

Velocity $v$

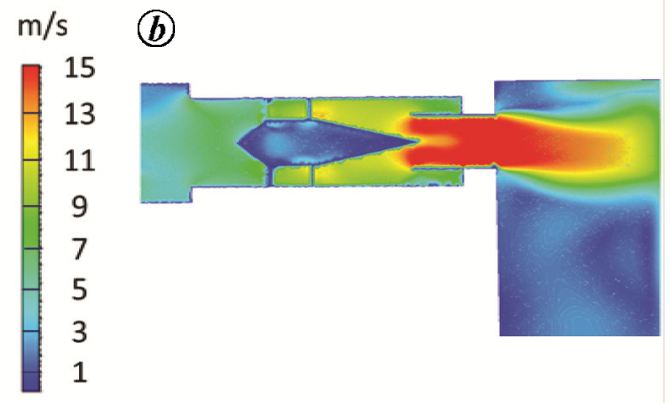

Velocity $v$

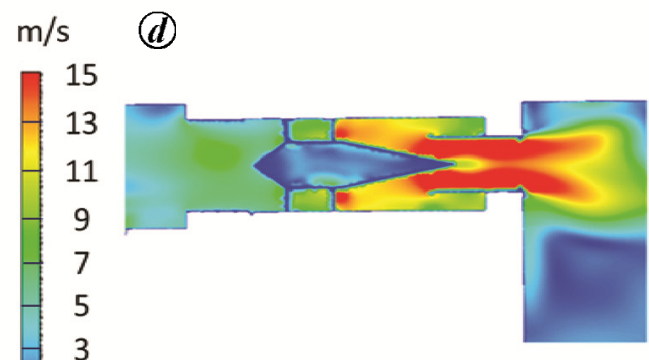

Figure 4. Velocity of gas-particle flow when $L_{1}=0.3 D(\boldsymbol{a}), 0.6 D(\boldsymbol{b}), 0.7 D(\boldsymbol{c}), 0.75 D(\boldsymbol{d})$ on the central surface of the plane $x z$.
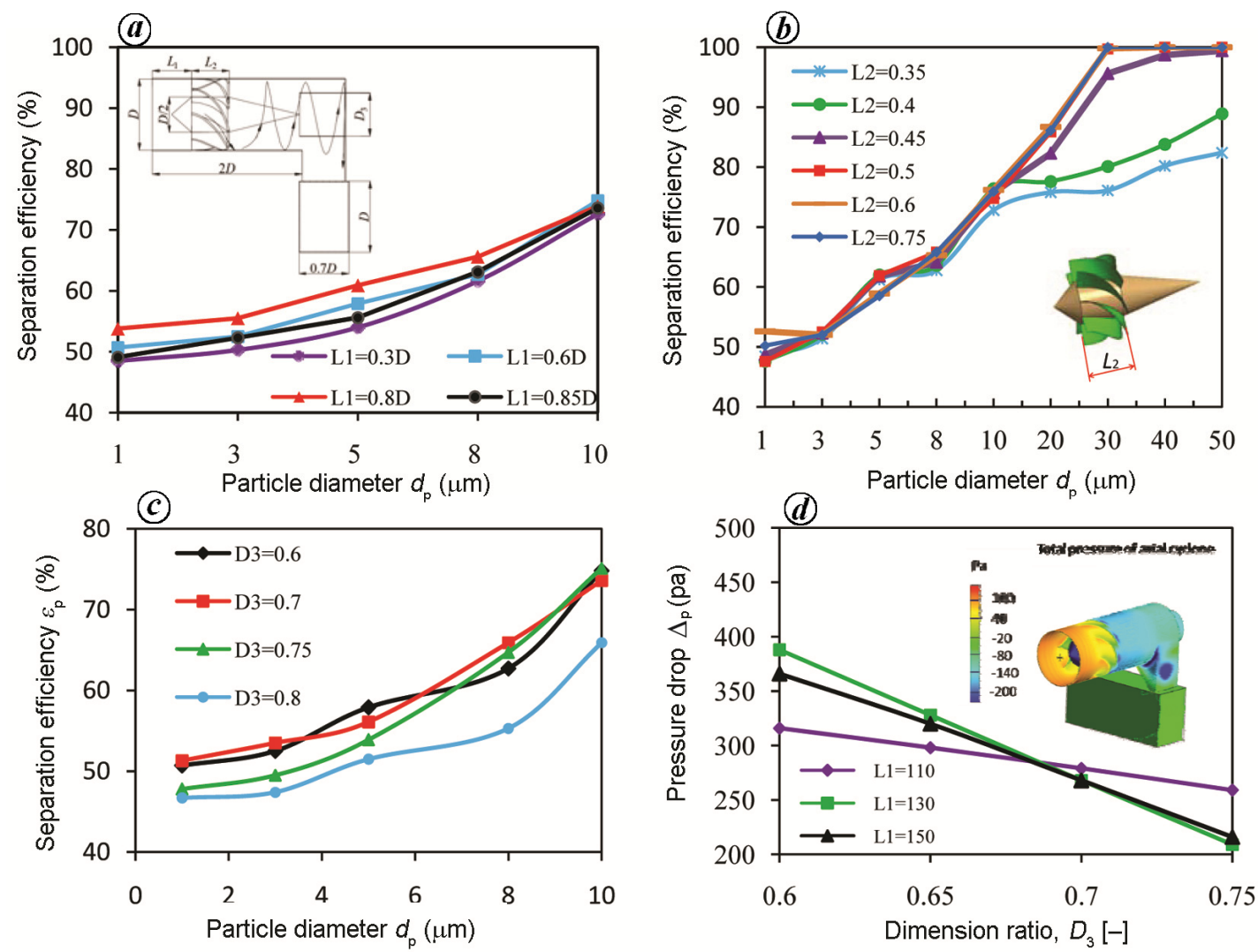

Figure 5. Influence of geometrical dimensions on separation efficiency and pressure drop. a, Effect of blade installation distance to inlet. $\boldsymbol{b}$, Effect of blade length. $\boldsymbol{c}$, Effect of outlet diameter. $\boldsymbol{d}$, Effect on pressure drop. 

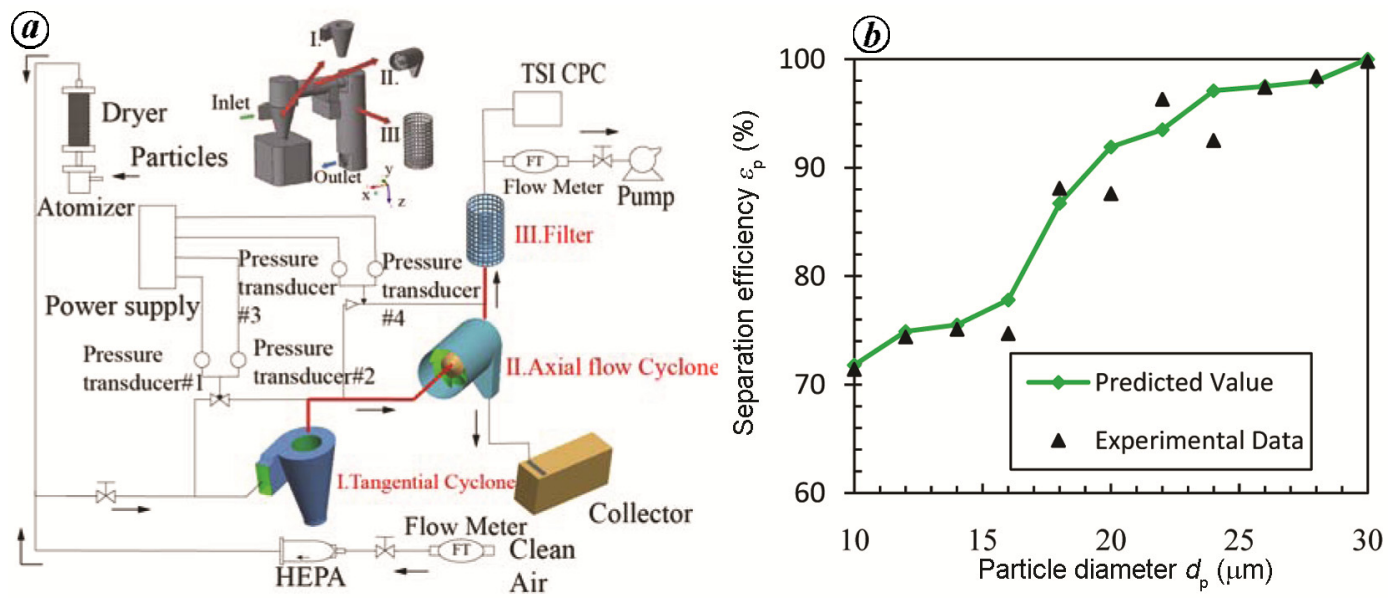

Figure 6. Experimental validation. $\boldsymbol{a}$, Experimental set-up. $\boldsymbol{b}$, Comparison of experimental data with the predicted collection efficiency.

the declining trend of $\Delta P_{\mathrm{a}}$ with increased $D_{3}$. But the slopes of the linear relationship between $\Delta P_{\mathrm{a}}$ and $D_{3}$ are not same when $L_{1}$ is different. This conclusion could be used for building air purifiers. The relationship between $\Delta P_{\mathrm{a}}$ and $D_{3}$ can be expressed by the equation of $\Delta P_{\mathrm{a}}=A$ $D_{3}+B$, where $A$ and $B$ are constants when $D_{3}$ is decided. In this simulation, the numerical values are $A_{1}=-370[-]$ and $B_{1}=538 \mathrm{~Pa}$ (when $L_{1}$ is $0.55 D$ ), $A_{2}=-980$ [-] and $B_{2}=954 \mathrm{~Pa}$ (when $L_{1}$ is $0.65 D$ ), $A_{3}=-800 \quad[-]$ and $B_{3}=868 \mathrm{~Pa}$ (when $L_{1}$ is $0.75 D$ ). Different cyclone separator models have different values of $A$ and $B$ and relevant research is needed for further simulations.

There are some advantages due to the linear relationship between $\Delta P_{\mathrm{a}}$ and $D_{3}$. (i) Through numerical simulation, we can find out the constant values of $A$ and $B$ in a special structure axial flow separator. The traditional method is that the structure designer can only estimate $\Delta P$ by measuring the relevant dimensions. (ii) The energy of the axial flow separator is provided by a pump. More $\Delta P_{\mathrm{a}}$ needs a more powerful pump. $\Delta P_{\mathrm{a}}$ can be calculated by the numerical simulation proposed by this study, and the specification of the pump can be estimated.

\section{Experimental validation}

Figure 6 is a schematic diagram of the experimental set-up used in the present study. Beech hardwood particles of sized $1-50 \mu \mathrm{m}\left(\rho_{\mathrm{w}}=800 \mathrm{~kg} / \mathrm{m}^{3}\right)$ were measured by an electronic scale, inhaled by a atomizer, dried by a silica gel drier, and finally controlled rate by a flowmeter ${ }^{19}$. As the particle flow generated by the atomizer is far less than the working flow required by the multi-stage dust collector, air is added to the inlet airflow after passing through the filter. A scanning mobility particle sizer (TSI model) was used to measure the particle size distributions at the end part of the cyclone collector. The escaped particles of each size were then measured by the measured particle concentrations $\left(\rho_{\mathrm{p}}\right)$ upstream and downstream of the collector.

In order to verify the simulation results, the numerical results are compared with the experimental data in Figure 13 , which shows the $\varepsilon_{\mathrm{p}}$ and $\Delta P$ of wood particles in the range of $d_{\mathrm{p}}=10 \mu \mathrm{m}$ to $30 \mu \mathrm{m}$. The maximum relative simulation error for $\varepsilon_{\mathrm{p}}$ is $e_{\max }=4.6 \%$, the average error of simulation for $\varepsilon_{\mathrm{p}}$ is $e_{\mathrm{ev}}=1.6 \%$.

\section{Conclusion}

A multistep dust collector has been studied using the SST model. The collector has higher separation efficiency $\varepsilon_{\mathrm{p}}$ than the individual tangential cyclone or the axial cyclone collectors for the same inlet conditions. The simulation results were verified by experiments under industrial conditions. Reasonable agreement was achieved in the comparison of separation efficiency between the experimental data and predictions, with a maximum error $e_{\max }=4.6 \%$. Thus, the numerical model proposed in this study is valuable for the optimum design and evaluation of the gas-particle two-phase flow industrial dust collector.

1. Bernardo, S., Mori, M., Peres, A. P. and Dionísio, R. P., 3-d computational fluid dynamics for gas and gas-particle flows in a cyclone with different inlet section angles. Powder Technol., 2006, 162, 190-200.

2. Chen, S., Liu, P. and Gong, J., Performance study of backflow type dynamic cyclone separator for coal-bed methane. Powder Technol., 2017, 305, 56-62.

3. Yao, J. and Takei, M., Application of process tomography to multiphase flow measurement in industrial and biomedical fields - a review. IEEE Sens. J., 2017, 17, 8194-8205.

4. Wan, G., Sun, G., Xue, X. and Shi, M., Solids concentration simulation of different size particles in a cyclone separator. Powder Technol., 2008, 183, 94-104.

5. Sun, X. and Yoon, J. Y., Multi-objective optimization of a gas cyclone separator using genetic algorithm and computational fluid dynamics. Powder Technol., 2017, 325, 347-360. 
6. Tan, F., Karagoz, I. and Avci, A., The effects of vortex finder dimensions on the natural vortex length in a new cyclone separator. Chem. Eng. Commun., 2016, 203, 1216-1221.

7. Duan, L., Wu, X., Ji, Z., Xiong, Z. and Zhuang, J., The flow pattern and entropy generation in an axial inlet cyclone with reflux cone and gaps in the vortex finder. Powder Technol., 2016, 303, 192-202.

8. Xue, X., Sun, G., Wan, G. and Shi, M., Numerical simulation of particle concentration in a gas cyclone separator. Pet. Sci., 2007, 4, 76-83.

9. Hsiao, T. C., Chen, D., Greenberg, P. S. and Street, K. W., Effect of geometric configuration on the collection efficiency of axial flow cyclones. J. Aerosol Sci., 2011, 42, 78-86.

10. Hsiao, T.-C., Chen, D.-R., Li, L., Greenberg, P. and Street, K. W., Development of a multi-stage axial flow cyclone. Aerosol Sci., Technol., 2010, 44, 253-261.

11. Chen, S. C. and Tsai, C. J., An axial flow cyclone to remove nanoparticles at low pressure conditions. J. Nanopart. Res., 2007, 9 , 71-83.

12. Wu, F., Huang, C., Chen, F., Zhang, W. and Jia, C., Experimental study on cyclone separator. In International Conference on Mechanic Automation and Control Engineering, Hohhot, China, 15-17 July 2011, pp. 2163-2166.

13. Menter, F. R., Kuntz, M. and Langtry, R., Ten years of industrial experience with the SST turbulence model. Turbulence, 2003, 4(1), 625-632.

14. Menter, F., Zonal two equation $k-\omega$ turbulence models for aerodynamic flows. In 23rd Fluid Dynamics, Plasma Dynamics, and Lasers Conference, Orlando, FL, USA, 6-9 July 1993.
15. Yoshida, H., Yang, K. S., Fukui, K., Akiyama, S. and Taniguchi, S., Effect of apex cone on particle classification performance of cyclone separator. J. Chin. Inst. Chem. Eng., 2004, 35, 41-46.

16. Liu, H., Wang, Y., Yuan, S., Tan, M. and Wang, K., Effects of blade number on characteristics of centrifugal pumps. Chin. J. Mech. Eng., 2010, 23, 742-747.

17. Ahmadpour, A., Abadi, S. M. A. N. R. and Meyer, J. P., Numerical investigation of pool boiling on a staggered tube bundle for different working fluids. Int. J. Multiphase Flow, 2018, 104, 89102.

18. Chen, J. and Shi, M., A universal model to calculate cyclone pressure drop. Powder Technol., 2007, 171, 184-191.

19. Hreiz, R., Lainé, R., Wu, J., Lemaitre, C., Gentric, C. and Fünfschilling, D., On the effect of the nozzle design on the performances of gas-liquid cylindrical cyclone separators. Int. J. Multiphase Flow, 2014, 58, 15-26.

ACKNOWLEDGEMENTS. This work was supported by the National Natural Science Foundation of China (Grant nos. 51706098, 11705143), Natural Science Foundation of Jiangsu Province (no. BK20170792) and Scientific Research Program Funded by the Shaanxi Provincial Education Department (no. 17JK0549), China.

Received 29 November 2018; revised accepted 22 February 2019

doi: $10.18520 / \mathrm{cs} / \mathrm{v} 117 / \mathrm{i} 12 / 1999-2005$ 